\title{
非晶 $\mathrm{Bi}_{4} \mathrm{Ge}_{3} \mathrm{O}_{12}$ 中原子近邻结构的 EXAFS 研究
}

\author{
沈志坚* 林云飞 陆坤权 赵雅琴 常龙存 \\ （武汉工业大学）（中国科学院物理研究所, 北京)
}

$\mathrm{Bi}_{4} \mathrm{Ge}_{3} \mathrm{O}_{12}(\mathrm{BGO})$ 晶体是一种理想的闪炼晶体, $\mathrm{Bi}^{3+}$ 为发光活性剂 ${ }^{[2]}$. 铋含量较高的铋锗 酸盐玻成由于其可能存在的闪柏性能引起人们的关注. 玻璃光学质量高、能够制成各种复杂 的形状,并且价格便宜,因此,除晶体材料外, 人们尝试用玻璃作为 $\mathrm{Bi}^{3+}$ 发光活性剂的基质, 这 是很自然的. 已有文献报道了铋锗酸盐玻璃中 $\mathrm{Bi}^{3+}$ 离子的光谱 ${ }^{[2]}$ 、衰变 ${ }^{[3]}$ 和能量转换 ${ }^{[4]}$ 性质,

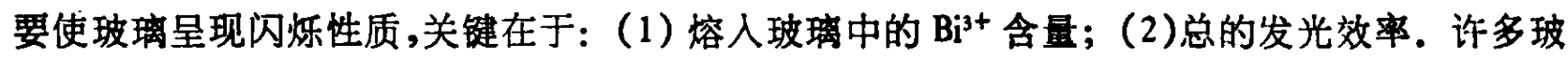
㻦中 $\mathrm{Bi}_{2} \mathrm{O}_{3}$ 的摩尔百分含量可以接近或超过 $\mathrm{BGO}$ 晶体中 $\mathrm{Bi}_{2} \mathrm{O}_{3}$ 的摩尔百分含量 $(40 \mathrm{~mol} \%)$ ， 但由于无序效应，玻璃中原子近邻结构可能与晶体中的不同，会影响 $\mathrm{Bi}^{{ }^{3+}}$ 离子的发光活性.

扩展X射线四收精细结构 (Extended X-ray Absorption Fine Structure, EXAFS)由于其 对吸收原子及吸收原子近邻结构的敏感性, 能直接有效地确定复杂体系非晶态物质中不同原 子的近邻结构参数, 如键长, 配位数等, 是近年来发展起来的一种确定非晶态物质中原子近邻 结构的有效方法. 本文报道 EXAFS 研究非晶 BGO 中 Bi, Ge 两种原子近邻结构的结果。

\section{一、实验}

实验采用的非晶 BGO 样品是相应组分的单晶经 $1200{ }^{\circ} \mathrm{C} 、 1 \mathrm{~h}$ 熔化后水淬得到的.EXAFS 测试在 RU-1000 (60kw) 转软 X 光机 (Rigaku Denki Co.) 和普通衍射仪改装的系统上完 成. 实验测定了非晶 BGO 中 Bi 原子的 LIII 吸收和 Ge 原子的 K 吸收 EXAFS 谱, 用 $\mathrm{s}^{\mathrm{i}}$ (422) 平晶单色器, Mo 知, 管流、管压分别为 $50 \mathrm{kV}$ 和 $400 \mathrm{~mA}$. 采用正比计数器和内柏计数管 组成的双探测器装冝及脉冲高度甄别系统, 成功地消除了X射线谱中特征线及晶体单色器高 次衍射产生之高次谐波的干扰后,得到的 EXAFS 谱是较为理想的.

图 1(a) 和 (b) 分别显示了实验测得的非晶 BGO 中 $\mathrm{Bi}, \mathrm{Ge}$ 两种原子的特征吸收谱, 相应 的 EXAFS 谱示于图 1(c) 和 (d). 采用通常的 EXAFS 数据处理程序, 将 EXAFS 数据作傅 里叶变换得到经向结构函数, 进行傅里叶滤波获得单配位层的结构信息, 通过与晶态标样 (这 里选 $\mathrm{GeO}$ 和 $\mathrm{BGO}$ 晶体)的比较, 求得非晶 $\mathrm{BGO}$ 中 $\mathrm{Bi}, \mathrm{Ge}$ 两种原子的近邻结构参数.

\section{二、实验结果与讨论}

1. Bi 原子的近邻结构

测定 $\mathrm{Bi}$ 原子的近邻结构时, 以 $\mathrm{BGO}$ 晶体为标样. $\mathrm{BGO}$ 晶体与天然硅铋矿 $\mathrm{Bi}_{4} \mathrm{Si}_{3} \mathrm{O}_{12}$ 同 晶型 ${ }^{[5]}$, 具有立方结构, 晶格常数为 $10.495 \AA$, 晶体中 Ge 原子与最近邻的四个 $O$ 原子配位成

本文 1986 年 7 月 11 日收到.

* 现在工作单位: 上海建材学院材料系. 

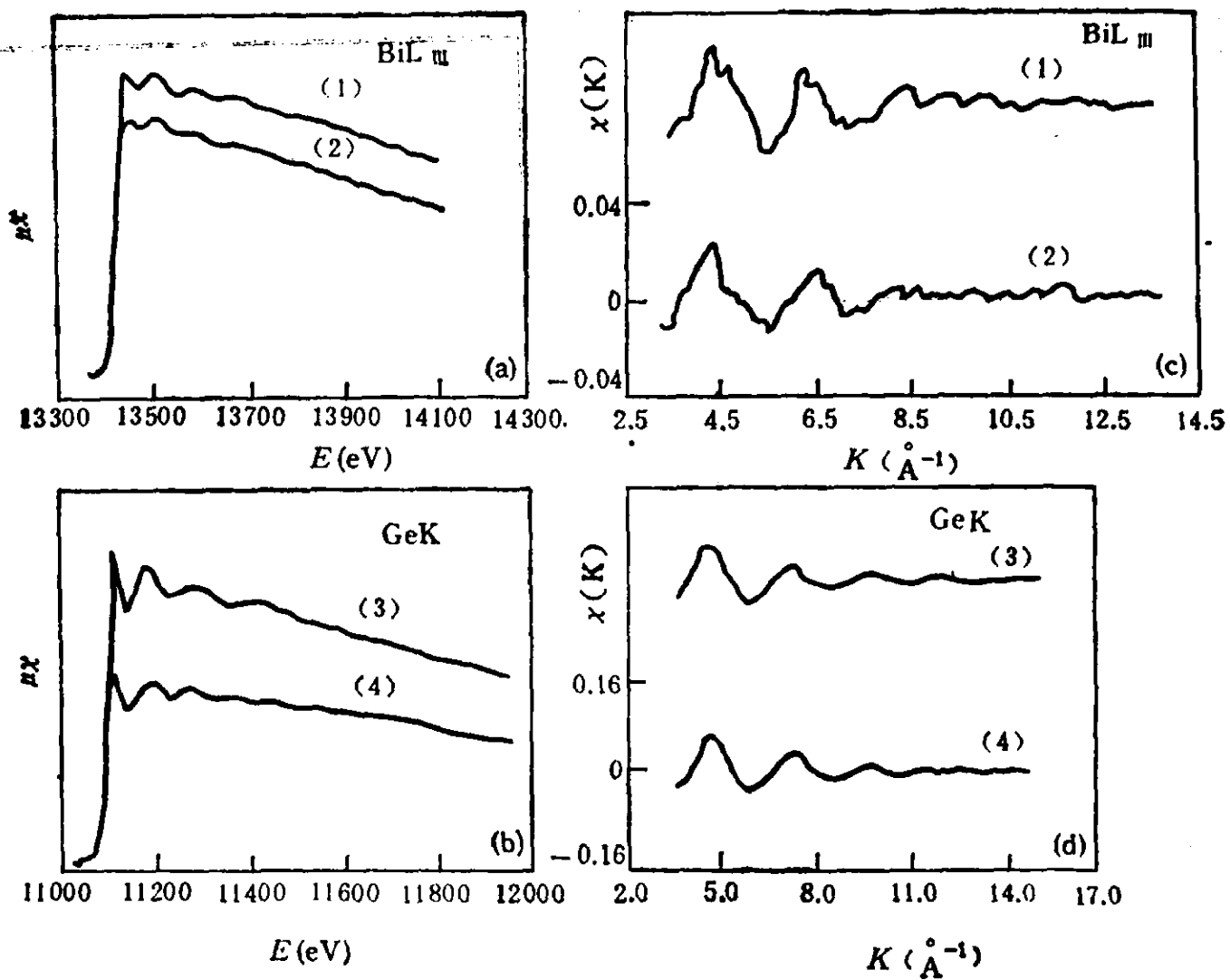

图 1 非晶 $\mathrm{Bi}_{4} \mathrm{Ge}_{3} \mathrm{O}_{12}$ 中原子的 $\mathrm{X}$ 射线特征吸收谱与晶态 $\mathrm{BGO}$ 以及 $\mathrm{GcO}_{2}$ 中的相比较

(a) Bi 的 LIII 吸收谱; (b) Ge 的K吸收谱; (c)、(d) 相应的 EXAFS 振荡.

(1) 晫态 $\mathrm{BGO}$; (2) 非晶 $\mathrm{BGO}$; (3) 晶态 $\alpha$-石英型 $\mathrm{GeO}_{*}$; (4) 非晶 $\mathrm{BGO}$

I $\left.\mathrm{GeO}_{4}\right]$ 四面体, 每个 $\mathrm{Bi}^{3+}$ 为六个 [ $\left.\mathrm{GeO}_{4}\right]$ 四面体所环绕, 每个 $\left[\mathrm{GeO}_{4}\right]$ 四面体顶角上的- - 个 $\mathrm{O}^{2-}$ 与 $\mathrm{Bi}^{3+}$ 相联, 使 $\mathrm{Bi}^{3+}$ 与 $\mathrm{O}^{2-}$ 形成一个变形的八面体，其中三个 $\mathrm{O}^{2-}$ 与 $\mathrm{Bi}^{3+}$ 的间距是 2.19 $\AA$, 而另三个 $\mathrm{O}^{2-}$ 与 $\mathrm{Bi}^{3+}$ 的间距为 $2.67 \AA$.

$\mathrm{Bi}$ 原子的径向结构函数示于图 2, 由图可以清楚地区分出 BGO 晶体中 Bi 原子最近邻的 二个配位层, 第一配位层六个氧原子分成不等距的两组, 在图上也可以清楚地看到. 与 BGO 晶体不同, 非晶 BGO 中 Bi 的径向结构函数上只能观察到 Bi 原子最近邻的一个配位层, 该配 位层对应一个对称的峰,计算得, Bi-O 间距为 $2.18 \AA$, 相应的配位数为 3 . 这一结果表明: 非 晶 $\mathrm{BGO}$ 中 $\mathrm{Bi}$ 的近邻结构与晶体中的不同, 晶体中不规则的 [ $\mathrm{BiO}_{6}$ ] 八面体在玻璃中产生了 更严重的畸变,变形的结果, 非晶 $\mathrm{BGO}$ 中 $\mathrm{Bi}$ 与O形成了类似 $\left[\mathrm{AsO}_{3}\right] 、[\mathrm{SbOs}]$ 的 $\left[\mathrm{BiO}_{3}\right]$ 基 团. $\left[\mathrm{BiO}_{3}\right]$ 易加人玻玹网络, 这也许正是玻璃中 $\mathrm{Bi}_{2} \mathrm{O}_{3}$ 含量可以较高的原因, 但玻璃中 $\mathrm{Bi}^{3+}$ 的近邻结构与晶体中的不同, 这会影响 $\mathrm{Bi}^{\mathrm{i}}$ 的发光活性.

\section{Ge 原子的近邻结构}

为了估计实验室 EXAFS 装置测键长的精确度, 我们测定了晶态和非晶态 $\mathrm{GeO}_{2}$ 中 $\mathrm{Ge}$ 原 子的近邻结构, 结果示于图 3. 晶态 $\mathrm{GeO}_{2}$ 有两种晶型, 金红石型和 $\alpha$-石英型, 在前一种晶型 中 $\mathrm{Ge}$ 原子与最近邻的六个 $\mathrm{O}$ 原子配位形成 $\left[\mathrm{GeO}_{6}\right]$ 八面体,在后一种晶型中 $\mathrm{Ge}$ 原子与最近 邻的四个 $\mathrm{O}$ 原子配位成 $\left[\mathrm{GeO}_{4}\right]$ 四面体. 非晶 $\mathrm{GeO}_{2}$ 中 $\mathrm{Ge}$ 原子的近邻结构与晶态 $\alpha$-石英型 $\mathrm{Ge}_{2} \mathrm{O}_{2}$ 中的相类似, 这在图 3 中可以清楚地分辩出. 以 $\alpha$-石英型 $\mathrm{GeO}_{3}$ 为标样, 计算得金红 石型 $\mathrm{GeO}_{2}$ 和非晶 $\mathrm{GeO}_{2}$ 中 $\mathrm{Ge}-\mathrm{O}$ 间距及配位数，与 $\mathrm{X}$ 射线衍射 ${ }^{[6]}$ 及中子衍射实验 ${ }^{[3}$ 所得的 


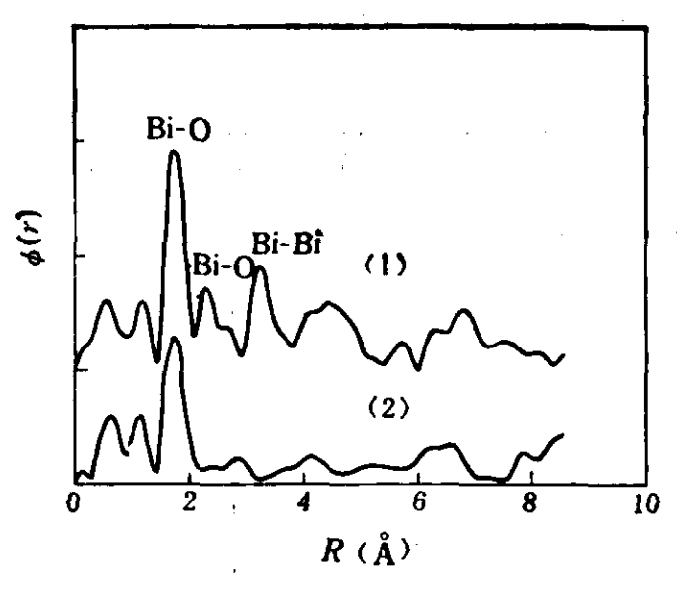

图 $2 \mathrm{Bi}_{4} \mathrm{Ge}_{3} \mathrm{O}_{12}$ 晶体 (1) 和非晶 (2) 中 Bi 原子的径向结构函数

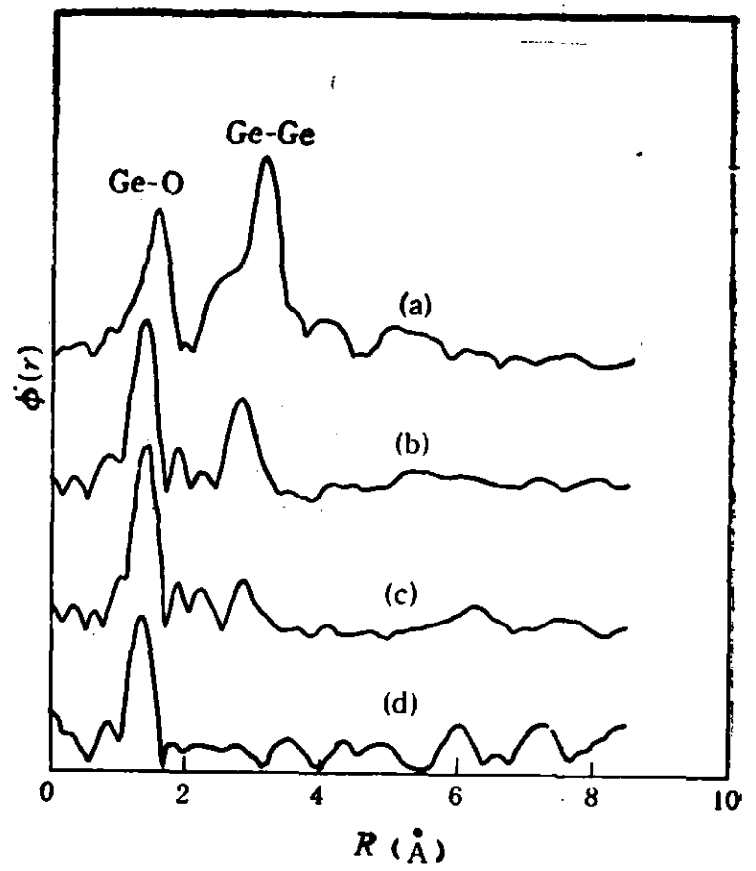

图3 非晶 $\mathrm{Bi}_{4} \mathrm{Ge}_{3} \mathrm{O}_{12}$ 中 $\mathrm{Ge}$ 原子的径向结构函数与 晶态及非晶态 $\mathrm{GeO}_{2}$ 中 $\mathrm{Ge}$ 原子的径向结构函数相比较
(a) 金红石型晶态 $\mathrm{GeO}_{2}$;
(b) $\alpha$-石英型晶态 $\mathrm{GeO}_{2}$;
(c) 非晶 $\mathrm{GeO}_{2}$;
(d) 非昆 $\mathrm{Bi}_{4} \mathrm{Ge}_{3} \mathrm{O}_{12}$

结果非常一致,与已发表的 EXAFS 研究结果也是相同的 ${ }^{[8]}$.

图 3(d) 为非晶 BGO 中 Ge 原子的径向结构函数, 由图可见, 非晶 BGO 中 Ge 原子的第 一配位近邻与非晶 $\mathrm{GeO}_{2}$ 以及 $\alpha$-石英型 $\mathrm{GeO}_{3}$ 晶体中 $\mathrm{Ge}$ 原子的第一配位近邻是类似的, 与 金红石型 $\mathrm{GeO}_{2}$ 晶体中 $\mathrm{Ge}$ 原子的第一配位近邻相差甚大. 计算得, 非晶 $\mathrm{BGO}$ 中 $\mathrm{Ge}-\mathrm{O}$ 间 距为 $1.75 \AA, \mathrm{Ge}$ 与最近邻的四个 $\mathrm{O}$ 原子配位成 [ $\mathrm{GeO}_{4}$ ] 四面体. 在图 3 所示的径向结构上还 可以观察到 $\alpha$-石英型 $\mathrm{GeO}_{2}$ 晶体、 $\mathrm{GeO}_{2}$ 非晶中都存在着 $\mathrm{Ge}-\mathrm{Ge}$ 配位的第二配位壳层, 但非 晶 BGO 中该配位峰消失了, 说明其中 $\left[\mathrm{GeO}_{4}\right]$ 四面体网络发展是不充分的, 同相应的晶体一 样, 非晶 $\mathrm{BGO}$ 中 $\left[\mathrm{GeO}_{4}\right]$ 四面体相互间是孤立的.

致谢: 人工晶体研究所韩艺捏同志提供了 BGO 单晶样品,喵致谢意.

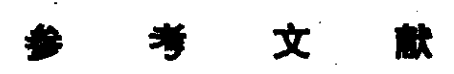

[1] Nestor, O. H. and Huang, O. Y, IEEE Trans. Nucl. Sci, NS-22(1975), 68.

[ 2 ] Boulon, G., Moine, B. and Bourcet, J. C., Phys. Rev., B22(1980), 1163.

[ 3 ] Boulon, G., Moine, B. and Bourcet, J. C. et al., J. Lumin, 18/19(1979), 924.

[ 4 ] Kalisky, Y., Reisfeld, R. and Boderheimer, J. S., J. Non-Cryst. Solids, 44(1981), 249.

[ 5 ] Segal, D. J., Santoro, R. P. and Newnham, R. E., Z. Krist., 123(1966), 73.

「6] Baur, W. H., Kahn, A. A., Acto Crystal., B27(1971), 2133.

[7] Wright, A. C., Leadbetter, A. J., Phys. Chem. Glasses, 17(1976), 122.

[ 8 ] Gaskell, D. H., Parker, J. M., The Structure of Non-Crystalline Materials. Taylor and Francis Ltd. London, New York, 1982, 42-52. 